\title{
Über die Gewebsatmung der Leber bei B=Avitaminose.
}

\author{
Von \\ Eishichiro Tsukamoto. \\ (家 本 䈋 七 郎) \\ (Aus der madianischen Klinik ron Prof. Dr. T. Kato \\ an der Tohoku Reichsuniversitut su Stndai.)
}

Ls ist schon von viclen Autoren darauf hingewicsen worden, dass bei Beriberi baw. B-Avitaminosis des Menschen, ausgenommen bei der Cardialform, der Grundumsatz meistens herabgesetzt ist, ferner dass auch bei Vögeln und Sïugern unter B-Vitaminmangel erniedrigter Gasumsatz sowohl des Gesamtorganismus als auch der einzelnen Organe nachzuweisen ist. Die Leber ist aber cin Organ, in welchem sich verschiedene lebenswichtige chemische Vorgänge, Oxydation, Reduktion, Spaltung und Synthese, lebhaft alspiclen; sic unterscheidet sich deshalb unter bestimmten Bodingungen manchmal in Bezug auf den Gasumsatz von anderen Organen, z. B. Muskel, Niere, Herz u. a. Jedenfalls darf man nicht ohne weiteres annehmen, dass bei B-Avitaminosis der Energiewechsel des Lebergewebes ganz dersclbe ist wie der der anderen Organgewobe.

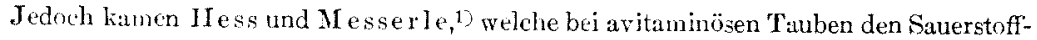
verbrauch verschiedener Gewebe mittelst Dinitrobenzol metho de ermittelten, zu dem Schluss, dass die Atmung der Leberzellen ebenso wie die der anderen Organzellen herah setzt ist. Abderhalden'2) ist aher der Ansicht, dass es sich bei der Farbstoffreduktion und dem Sauerstofferbrauch des avitaminösen Gewebes nicht um einen identischen Vorgang handelt, Mittelst des Krog h'schen Mikrorespirometers hat $\mathrm{R}_{0} \mathrm{ch} \mathrm{e}^{3}$ die $\Delta$ tmung der verschiedenen Organgewebe, insbesondere des Lebergewebes, bei avitaminösen Tauben und Müusen beobachtet und konstatiert, dass der Sauerstoffrerbrauch der Organe auch bei A vitaminosis nicht anders ist als in der Norm. Mi y a k et), der nach War burg scher Methode den Saluerstoffverbrauch des Lebergewebes on Reistaben bestimmte, lionnte gleichfalls keine erhebliche Iferabsetzung desselben nuchweisen.

1) Ifessu. Messerle, Zeitschr. f. physiol. Chem., 1922, 119, 176.

2) Abderhalden, Zeitschr. f. physiol. Chem., 1924, 134, 97 .

3) Roche, Arch. intern. d. Physiol, 1925, 24, 413.

4) Mi ake, Naibunpitsu-Gakkai-Zasshi, 1926, 2, 642. 
Bei dieser Divergenz der Boobachtungsresultate schien es mir von Belang, die Gewebsatmung, insbesondere die der Leber, bei B-Avitaminose nochmals zu untersuchen und zwar mittelst der Warburgschen Methode direkt nachzuprïfen, ob sie in irgendeinem Verhalten nicht ron dem der anderen Organe oder ïherhaupt von der Norm abweicht. Die Ergebnisse meiner Cntersuchung seien hier kurz mitgeteilt.

Untersuchungsmethode: Taube, die mit gut poliertem Reis gefüttert wurde und bereits (ungeführ zwei Wo hen nach Beginn der Fütterung) Symptome von B-Avitaminose darbot, lässt man durch Abschneiden des Halses verbluten und nimmt die Lober unter ascptischen Kautelen heraus. Von der Leber, deren Wasser mit sterilem Löschpapier sorgfältig abgetupft ist, schneidet man mit einem scharfen Rasiermesser dünne Schnittstücke ab, deren Dicke dünner als $1 \mathrm{~mm}$ und deren Gewicht unter $100 \mathrm{mg}$ sein kann, bringt sic in ein Atmungskölbchen, in welchem die nötige Menge von Ringerlösung und Natronlauge enthalten ist, und misst den Saucrstoffverbrauch der Shnitte nach Warburgsther Nethode ${ }^{5 i)}$ in einem anf $37^{\circ} \mathrm{C}$ gehaltenen Thermostat. Das Kölbehen wird 80 oder $90 \mathrm{mal}$ pro Minute in dem Wasserbad geschüttelt, die Manometerskala des Apparats meistens alle zehn oder zwanzig Minuten abgelesen. Auf dicse Weise beobachtete ich 60 bis 120 Minuten lang den Verlauf des Sanerst ffverbranchs der Shnitte. Die Kontrollversuche wurden an der Leber gesunder, mit unpolierter Gerste gefütterter Tauben ausgeführt.

\section{Versuchsresultate.}

Tab. 1 zeigt den Sauerstoffverbrauch des Lebergewebes von normalen Tauben. Nach ihr jst die Atmungsgrösse der Gewebsstücke individuell gewissermassen verschieden, der maximale Wert betrügt $5774 \mathrm{cmm}$, der minimale $4282 \mathrm{cmm}$ und der Mittelwert $4818 \mathrm{cmm}$ prog pro Stunde.

Der Sauerstoffverbrauch der avitaminösen Lebergewebe ist in Tab. 2 wiedergegeben. Man ersieht aus der Tabelle, dass das Lebergervebe der Reistauben mit manifesten a vitaminösen Sy mptomen deutlich hırabgesetzten Siuerstoffverbrauch aufweist, obwohl dieser wie bei normalem Lebergewebe etwas individueller Schwankung unterliegt.

Da aber die an Avitaminose erkrankte Taube, wie aus Tab. 2 ersichtlich, auffillende Körpergewichtsabnahme bekommt, crhebt sich die Frage,

5) Warburg, Abderbalden: Handbuch d. biol. Arbeitsmethode. IV, Be:lin und Wien $1929,251$.

6) Warburg, Biocbem. Zeitschr., 1923, 142, 317. 
Tabelle 1.

\begin{tabular}{|c|c|c|}
\hline Vers. Nr. & Körpergewicht (g) & Og-Verbrauch (cmnt pro g pro Std.) \\
\hline 1 & 220 & $454 i$ \\
\hline 2 & 240 & 4283 \\
\hline$\because$ & 240 & 4721 \\
\hline 4 & 270 & 4893 \\
\hline 5 & 290 & 4510 \\
\hline 6 & 260 & 4861 \\
\hline 7 & 390 & 4638 \\
\hline 8 & 280 & 4686 \\
\hline 9 & 280 & 5260 \\
\hline 10 & 260 & 5774 \\
\hline 11 & 240 & 4896 \\
\hline 12 & 810 & 5080 \\
\hline \multirow{2}{*}{\multicolumn{2}{|c|}{ Mittelwert }} & 4985 \\
\hline & & 4818 \\
\hline
\end{tabular}

Tabelle 2.

\begin{tabular}{|c|c|c|c|c|}
\hline Vers, Nr. & $\begin{array}{l}\text { körpe } \\
\text { vor u. nut } \\
\text { vor }\end{array}$ & $\begin{array}{l}\text { ht }(g) \\
\text { ranktung } \\
\text { nach }\end{array}$ & $\begin{array}{c}\text { Dauer d. Fitterung } \\
\text { (Tage) bis zur } \\
\text { Leberentnahme }\end{array}$ & $\begin{array}{c}\text { O.-Verbrauch } \\
\text { (cmm prog pro Std.) }\end{array}$ \\
\hline $\begin{array}{c}1 \\
3 \\
3 \\
4 \\
5 \\
6 \\
7 \\
5 \\
9 \\
10 \\
11 \\
12 \\
13 \\
14\end{array}$ & $\begin{array}{r}290 \\
290 \\
800 \\
260 \\
240 \\
260 \\
230 \\
270 \\
260 \\
280 \\
260 \\
280 \\
250 \\
250 \\
\text { Tittelwert }\end{array}$ & $\begin{array}{l}230 \\
230 \\
280 \\
280 \\
190 \\
180 \\
240 \\
180 \\
200 \\
180 \\
200 \\
190 \\
190 \\
160 \\
190\end{array}$ & $\begin{array}{l}26 \\
25 \\
28 \\
24 \\
24 \\
23 \\
28 \\
18 \\
19 \\
20 \\
21 \\
21 \\
21 \\
24 \\
25\end{array}$ & $\begin{array}{l}4070 \\
4124 \\
4019 \\
4001 \\
4105 \\
4051 \\
3686 \\
3598 \\
3440 \\
3686 \\
4089 \\
3397 \\
4183 \\
3650 \\
3864\end{array}$ \\
\hline
\end{tabular}

Tabelle 3.

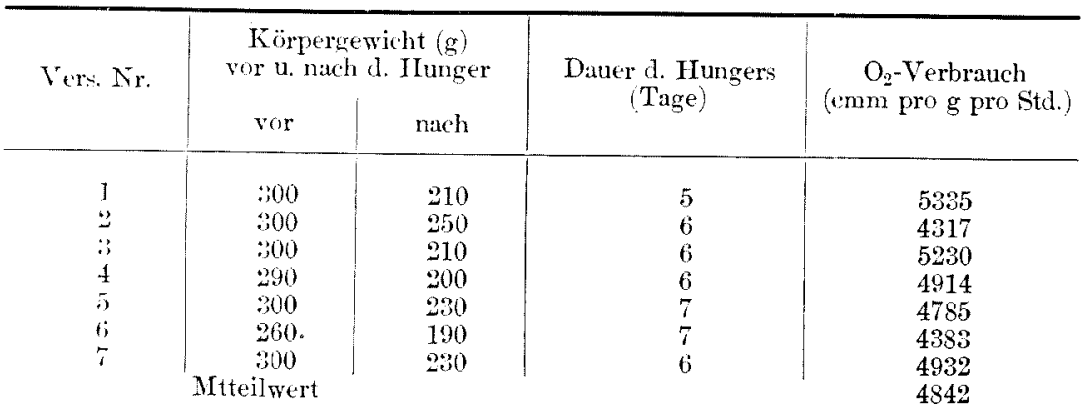


ob es sich beim verminderten Sanerstotfverbrandh der avitaminösen Taubenleber nur um eine Folgecrsheinung dor einfuchen Inanition, nicht um ein eigentliches avitaminöres Symptom handelt. Un dicse Frage zu beantworten, habe idh in einer anderen Versuchsreihe den Sancrsteffrerbrauth des Lebergeweles der 'Tatuben gemessen, weldhe mit Wanser allecin oder mit ganz mangellatten Nahrungsstoffen gefüttert wurden, infolgedessen stark abmagerten in gledom Masso wie lle avituminüsen Tauben in ihrem Körpergewielı reluziert waren. 'Tab. "3 stcllt das Ergebnis dar.

Verglicht man die drei Tabellen, so ist leicht zu crehen, lass der Satuerstofferbratuch des I ebergewebes bei Tauben, weldhe durch Hunger stark abgemagert sind, fast gleidh ist wie ler der Kontrolle und weit grösser als der bei B-Avitaminose. Daraus geht hervor, dass die Herabetzung des Sauerstotfverbrauh des avitaminösen Lubergewebes nicht durch einfachen Ernahrungsmanged belingt ist. Gross' und Nenhaus' Befund, dass die Gewebsatmung der Rattenleber bei Hunger nicht von der Form abweicht, steht mit dem Resultat moines Hungerversuhes gut im Einklang.

$$
\text { Zusammenfassung. }
$$

1. Bei der durch Fütterung von pulicrtem Reis crzengten Avitaminosis der Taube ist der Saucrsteff verbrameh des I debergewebes auffallend herabgesetzt.

2. Bei dem durch Hunger hervorgerufinen einfichen Ernährungsmangel zeigt die Atmungsgrösse der Taubenleber keine Abweichung von der Norm.

7) Grossu. Neuhaus, Peitr. z. path. Anat. u. allg. Path., 1927, 77, :304. 